\title{
Aktuelle
}

\section{Therapieergebnisse \\ und Perspektiven mit Bendamustin}

\section{Bendamustin-Gipfeltreffen}

Spitzingsee, 10.-11. März 2001 
B endamustin ist ein alkylierendes Zytostatikum, das zusätliche A ktivität als P urinanalogon aufweist. D ie Substanz ist nachweislich wirksam beim M ultiplen $M$ yelom, bei verschiedenen soliden Tumoren und bei $\mathrm{N}$ on-H odgkinLymphomen ( $\mathrm{NHL}$ ), insbesondere den niedrig malignen Formen. A ufgrund der ausgezeichneten Verträglichkeit wird B endamustin in der Palliativtherapie anderen Z ytostatika vorgezogen.

\section{Diagnostik niedrig maligner NHL}

D ie U nterscheidung der Lymphom-U ntergruppen gestaltet sich oft sehr schwierig. Mit der neuen REA L-Lymphomklassifikation wird die Identifizierung bestimmter Subtypen jedoch erleichtert. Sie beinhaltet zusätzlich zu den rein morphologischen Kriterien immunotypische molekulare $\mathrm{M}$ arker, die den A utoren älterer Klassifikationen noch nicht zur Verfügung standen.

D as A usmaß der Schwierigkeiten bei der Typisierung wird durch eine Studie illustriert, in der Pathologen sechs deutscher $R$ eferenzzentren mit herausragender Expertise, die an der REA L-K lassifikation mitgearbeitet haben, Präparate von Primärpathologen beurteilten. Bei NHL, die von den Primärpathologen als $M$ antelzell-Lymphome diagnostiziert worden waren, konnte die Diagnose lediglich in einem D rittel der Fälle bestätigt werden. U mgekehrt wurden von allen Lymphomen, die von den R eferenzpathologen als $M$ antelzell-Lymphome diagnostiziert worden waren, nur ein D rittel von den Primärpathologen als solche erkannt. Daraus schließt M artin D reyling, M ünchen, dass es sehr schwierig ist, die Diagnose eines bestimmten niedrig malignen Lymphoms allein nach morphologischen K riterien zu treffen.

Der Typ des Lymphoms bestimmt entscheidend die Prognose und vielfach auch dieA uswahl der Therapie. B ei niedrig malignen $\mathrm{NHL}$ bestehen $U$ nterschiede von $40-80 \%$ in den 5-Jahres-Ü berlebensraten. B eim $M$ antelzell-Lymphom ist die Lebenserwartung noch wesentlich geringer. Daraus schließt D reyling, dass die Unterscheidung von hoch und niedrig malignen $\mathrm{NHL}$ dem aktuellen Wissensstand nicht mehr entspricht. Durch die neuen molekularen Marker sind zusätzliche Prognosekriterien dazu gewonnen worden, die zur optimalen Behandlung des Patienten auch bestimmt werden sollten.

M antelzell-L ymphom

In der REA L-K lassifikation wird diese N eoplasie als zentrozytisches $L$ ymphom bezeichnet. E szeigt niedrig maligne M orphologie und geringe Proliferationsrate. Charakteristisch ist die Translokation t(11;14), die zu erhöhter Produktion von Zyklin D 1 führt. Letzteres sichert die Diagnose: Es gibt kein Mantelzell-Lymphom ohne Zyklin-D 1-Ü berexpression.

O bwohl das M antelzell-Lymphom morphologisch zu den niedrig malignen Lymphomen gezählt wird, verhält es sich im klinischen Verlauf eher aggressiv. Die mittlere Ü berlebenszeit bestätigter $M$ antelzell-Lymphome liegt bei etwa 3 Jahren. E ine optimale Therapie dieses Lymphoms ist noch nicht definiert. Zirka 400 Fälle sind weltweit retrospektiv ausgewertet worden, überwiegend wurden COP (Cyclophosphamid, Vincristin, Prednison) bzw. CH OP (Cyclophosphamid, Doxorubicin, Vincristin, Prednison) appliziert, wobei unklar ist, ob R egime mit A nthrazyklin von Vorteil sind. M an findet maximal $40 \%$ komplette Remissionen, aber keine Verlängerung des progressionsfreien Ü berlebens und des $\mathrm{G}$ esamtüberlebens durch konventionelle Chemotherapien.

In Studien zur Hochdosischemotherapie mit autologer Stammzelltransplantation beim $\mathrm{M}$ antelzellLymphom fand man 2-Jahres-Ü berlebensraten zwischen 16 und 100\%. Die hohe Schwankungsbreite resultiert aus unterschiedlichen Kriterien bei der Patientenselektion. O ffenbar lassen sich bei bestimmten Patientengruppen hohe R emissionsraten erzielen. A us diesem G rund hat sich die deutsche Studiengruppe einer europäischen Studie angeschlossen, die das H ochdosiskonzept als I nitialtherapie des $\mathrm{M}$ antelzell-Lymphoms testet. M ittlerweile sind 170 Patienten rekrutiert. Die H ochdosischemotherapie wird einer Interferon-E rhaltungstherapie gegenübergestellt.

In einer anderen Studie an Patienten mit M antelzellLymphom und anderen therapieresistenten $\mathrm{NHL}$ wurde, nach initialer $\mathrm{Chemotherapie,} \mathrm{eine} \mathrm{kombinierte}$ $\mathrm{R}$ adio-Chemotherapie ( $\mathrm{G}$ anzkörperbestrahlung) mit Rückgabe der zuvor gesammelten Stammzellen durchgeführt. Der Vergleichsarm der Studie war ebenfalls I nterferon-E rhaltungstherapie.

D ie A uswertung ergab einen frühzeitigen, signifikanten U nterschied hinsichtlich der rezidivfreien Ü berlebenszeit, aber keine Verlängerung des $\mathrm{G}$ esamtüberlebens. N egative Spätwirkungen sind sekundäre hämatologische $\mathrm{N}$ eoplasien. $\mathrm{D}$ ie I nzidenz des myelodysplastischen Syndroms beträgt 5-20\% nach 5 Jahren.

Interferon kann die progressionsfreie Ü berlebenszeit von 2,5 auf etwa 5 Jahre verlängern. E ine Indikation für Interferon besteht gerade bei therapieresistenten Lymphomen, wie z. B. dem M antelzell-Lymphom.

\section{Follikuläre Lymphome}

Diese stellen ein Viertel bis ein D rittel aller indolenten Lymphome. Sie sind charakterisiert durch die Translokation $\mathrm{t}(14 ; 18)$, q32; $\mathbf{2 1}$, durch die das an 
A poptosehemmung beteiligte bcl-2-G en transloziert und dereguliert wird. Die Translokation ist in etwa 80-90\% der Fälle nachweisbar. Auch bei diesen Lymphomen kommt es, obwohl morphologisch klassische zentrozytische und zentroblastische Elemente gut nachweisbar sind, außerhalb des Verbunds der Referenzpathologen nur in $60-70 \%$ zu richtigen D iagnosen.

D ie K rankheit wird häufig erst in den Stadien III und IV diagnostiziert. Es gibt keine zwingende Therapie, erst bei B eschwerden wird palliativ behandelt. In den wenigen Fällen (10-15\%), die früh erkannt werden, besteht die Möglichkeit kurativer lokaler Strahlentherapie.

D urch Palliativtherapie ist die G esamtüberlebenszeit nicht zu beeinflussen. Eine zurückhaltende orale Monotherapie bewirkt genau wie das aggressive CHOP-R egime 5-Jahres-Ü berlebensraten von $60 \%$. $D$ ies hat sich seit den 60er Jahren nicht geändert. $\mathrm{H}$ insichtlich der Z eitspanne progressionsfreien Ü berlebens sind jedoch Fortschritte gemacht worden. Purin-A naloga könnten bei rezidiviertem follikulärem Lymphom gängigen K ombinationen gleichwertig sein in Bezug auf A nsprechen und progressionsfreie Ü berlebenszeit. Komplette R emissionen sind selten. M öglicherweise kann man die PurinanalogaTherapie optimieren: Zuerst wird mit einer alkylierenden Substanz die DNA-R eparatur aktiviert, anschließend werden unter Purin-A naloga falsche Nukleotide eingebaut. Dieser theoretische Synergismus hat sich im klinischen A Iltag bestätigt.

A us ähnlichen Ü berlegungen entstand eine Phase-I/I IKonzeption, bei der in A nalogie zum M CP-R egime Chlorambucil durch Bendamustin ersetzt werden soll. Dies ist vielversprechend, weil Bendamustin neben seiner Eigenschaft als alkylierender Stickstofflostverbindung eine antizipierte Z usatzwirkung als Purin-A nalogon aufweist. Dies könnte sich als optimale Therapieoption für niedrig maligne Lymphome erweisen. Bei den ersten Patienten dieser Studie hat D reyling bereits E rfolge gesehen, $z$. B. bei einer Patientin mit Mantelzell-Lymphom, die nach 4 Zyklen mit dieser Kombination bereits in eine komplette R emission gekommen ist.

\section{Risikostratifikation bei chronisch lymphatischer Leukämie (CLL)}

B ertold $E$ mmerich, $M$ ünchen, berichtete über Konzepte aus der CLL-Studiengruppe.

$B$ esonders interessant ist die Definition von $\mathrm{R}$ isiko- faktoren bereits im Stadium Binet $A$, um frühe $\mathrm{K}$ rankheitsprogression vorhersagen zu können. D iese Fragestellung wird in der CLL-1-Studie untersucht. $B$ asis für dieses Konzept ist eine italienische Studie, in der eine $G$ ruppe von Patienten im Stadium A mit maximal 4J ahren Ü berlebenszeit abgegrenzt werden konnte [M olica et al., 1991]. N ormalerweise beträgt die G esamtüberlebenszeit von Patienten im Stadium A über 10 Jahre. Wenn es gelingt, diejenigen Patienten zu identifizieren, bei denen man ein rasches Fortschreiten der E rkrankung erwarten muss, kann man sie in einer frühen Phase der Erkrankung behandeln.

E infach zu bestimmende Serumparameter, die H inweise auf eine schlechte Prognose geben können, sind:

- nicht noduläre K nochenmarksinfiltration,

- Lymphozytenzahl über $50000 / \mu \mathrm{l}$,

- Lymphozytenverdopplungszeit unter 12 M onaten,

- $\beta_{2}$-M ikroglobulin über 3,5 ng/l,

- TK über $7 \mathrm{U} / \mu \mathrm{l}$,

- SCD 23 über $574 \mathrm{U} / \mu \mathrm{l}$.

$\mathrm{N}$ ach diesen $\mathrm{K}$ riterien werden $\mathrm{R}$ isikopatienten ermittelt und dann in die Gruppen «Behandlung mit Fludarabin» oder «B eobachtung» randomisiert. Z iel der Studie ist es, die Wirkung von Fludarabin im Stadium A und den Stellenwert der zell- und molekularbiologischen Parameter für die Prognose zu bestimmen. Vielversprechend ist der $\mathrm{N}$ achweis von p53-D eletionen in Chromosom 17 durch In-situ$\mathrm{H} y$ bridisierung mit Fluorescein-markierten DNA Sonden [D öhner et al., 1995].

Wenn die $U$ rsprungszellen einer $C L L$ naive $B$-Zellen sind, ist die Prognose in der R egel schlecht, sie beträgt 2-5 Jahre. Sind die U rsprungszellen dagegen M emory-B-Z ellen, so ist die Prognose gut, man kann mit einer Lebenserwartung von 10 Jahren rechnen [H amblin et al., 1999]. Zur U nterscheidung wird die genetische Feinabstimmung des variablen $\mathrm{A}$ nteils der G ene für die schwere I mmunglobulin-K ette (IgV H) nachgewiesen, die beim Übergang von naiven zu M emory-Zellen stattfindet.

Innerhalb der nächsten 2 Jahre wird man die Frage beantworten können, ob es bei CLL zwei Prognosegruppen gibt. In Kombination mit der Identifizierung bestimmter Chromosomenmutationen [D öhner et al., 2000] erhält man voraussichtlich noch genauere Informationen über die Prognose:

- Deletion 13q- 62\% (prognostisch günstig);

- Deletion 11q- 8\% (große Lymphadenopathie;

Prognose ungünstig und altersabhängig);

- Trisomie 12 11\%;

- Deletion 17p- 6\% (p53-Verlust; prognostisch ungünstig);

- D eletion 6q- 3\%. 


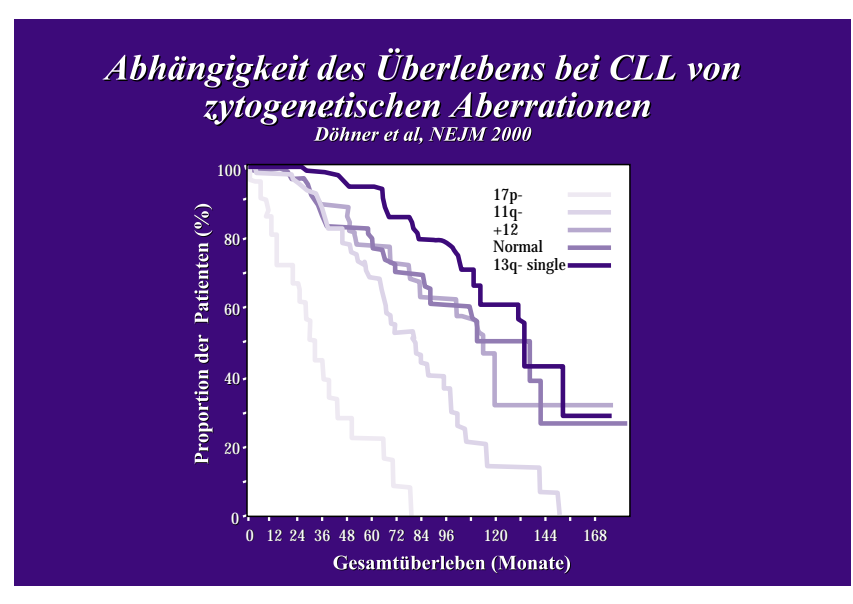

CLL mit den Deletionen $17 p$ - und $11 q$ - haben nach bisherigen Erfahrungen die schlechteste Prognose. E benfalls relevant sind die A ktivität der Laktatdehydrogenase ( $\mathrm{LDH}$ ) und die A nzahl der Leukozyten. Z usätzlich verschlechtert wird die Prognose, wenn die U rsprungszellen naive B-Zellen sind.

Zur U ntersuchung des Zusammenhangs von U rsprungszelltyp und Chromosomenaberrationen für die Prognose musste die Fallzahl der CLL-1-Studie auf 400 erhöht werden.

Die CLL-Studiengruppe hat inzwischen begonnen, auch $B$ endamustin systematisch zu untersuchen.

\section{Bendamustin-Monotherapie bei CLL}

Ohne Risikostratifikation richtet man sich bei CLL vielfach nach dem $A$ Iter: $F$ ür die J üngeren verwendet man in der R egel eine aggressivere $E$ rstlinien-Therapie; sehr alte, multimorbide Patienten werden vorerst nur beobachtet und dann, so schonend wie möglich, zunächst mit Chlorambucil behandelt.

Weniger klar sind die Strategien bei den anderen Patientengruppen, insbesondere jenen mit Kontraindikationen gegen bestimmte M edikamente: Fludarabin bei Autoimmunphänomenen oder anthrazyklinhaltige R egime bei kardialer Vorbelastung.

Bendamustin ist bei CLL aufgrund seines B-Zelldepletierenden $\mathrm{E}$ ffekts dem Cyclophosphamid klinisch überlegen [A nger et al., 1975].

In einer kürzlich publizierten Phase-II-Studie zu B endamustin bei $C L L$ [K ath et al., 2001] wurden je nach A Iter der Patienten $250-300 \mathrm{mg} / \mathrm{m}^{2}$ B endamustin pro Zyklus, verteilt auf 5 Tage, gegeben. Im Verlauf der Studie ergab sich aufgrund von L eukopenie die N otwendigkeit einer A ntibiotika-Prophylaxe, insbesondere gegen Pneumocystis-carinii-Pneumonie. Die Remissionsraten waren sehr hoch; 15 von 20 Patienten erreichten R emissionen, davon 6 eine komplette

\section{Ergebnisse zu Bendamustin-Monotherapie bei der CLL}

Referent

(Autor, Jahr) phase d. Pat.* handlung (in \%) (in \%) (in \%)

$\begin{array}{lllllll}\text { Anger, } 1975 & \text { III } & 39 & \text { nein } & 82 & 28 & 54\end{array}$

$\begin{array}{lllllll}\text { Blumenstengel, } 1997 & \text { II } & 16 & 1 / 16 & 68 & 18 & 50\end{array}$

$\begin{array}{lllllll}\text { Bremer, 1999 } & \text { II } & 15 & 15 / 15 & 94 & 7 & 87\end{array}$

$\begin{array}{lllllll}\text { Kath, } 2001 & \text { II } & 23 & 10 / 23 & 75 & 30 & 45\end{array}$

$\begin{array}{lllllll}\text { Aivado, } 2001 & \text { II } & 14 & 13 / 14 & 64 & 36 & 28\end{array}$

* Phase III-Studie: Pat. in Therapie-Arm mit Bendamusin

R emission. U nter L etzteren befand sich auch ein mit Fludarabin vorbehandelter Patient.

$M$ anuel Aivado, Düsseldorf, berichtete von seinen Daten einer Pilotstudie, die auf dem diesjährigen A SCO-Kongress anlässlich einer Postersitzung präsentiert werden. In einer Phase-II-Studie wurden Patienten mit teilweise mehrfach vortherapierter CLL mit Bendamustin in einer Dosierung von $100 \mathrm{mg} / \mathrm{m}^{2}$ an den Tagen 1+2, Wiederholung alle 4 Wochen, behandelt. A ntibiotika-Prophylaxe wurde bei zu kleiner T-H elfer-Zellzahl durchgeführt. $28 \%$ der Patienten erreichten eine PR, 36\% eine CR. In einer problematischen $U$ ntergruppe von 8 mit Fludarabin Vorbehandelten und 6 Patienten mit Autoimmunphänomenen kam es zu 3 kompletten, 2 partiellen Remissionen und 4 stabilen Erkrankungen. 1 Patient erlitt Progression im Kontext eines R ichterSyndroms. Die Therapie wurde ausgesprochen gut vertragen.

A ufgrund der hohen Wirksamkeit und der guten Verträglichkeit von B endamustin haben $\mathrm{A}$ ivado und $\mathrm{Kol}$ legen eine Folgestudie entworfen. In dieser Studie

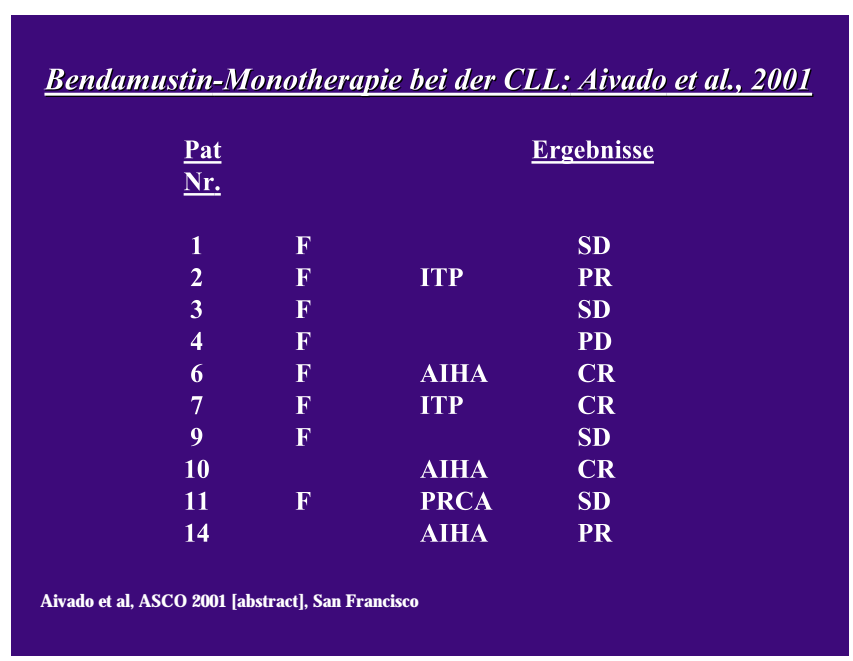


werden neben Wirksamkeit, Toxizität und immunphänotypischer Charakterisierung auch Chromosomenaberrationen, insbesondere p53-D eletionen, bewertet. $G$ rundlegende $E$ rkenntnisse erhofft man sich von den Genexpressionsprofilen der CLL-Patienten vor, während und nach Bendamustin-Therapie. D iese Profile werden mit der «Chip-Technologie» bestimmt. Dazu wird markierte RNA des Patienten mit 1200 verschiedenen, separaten $M$ arkergenen auf einer $\mathrm{N}$ ylon-M embran hybridisiert.

D ie A rbeitgruppe erhofft sich aus B endamustin-induzierten Variationen im $\mathrm{E}$ xpressionsmuster der $\mathrm{G}$ ene A nhaltspunkte für eine Stratifizierung von Patientengruppen, die für diese Therapie besonders gut bzw. nicht geeignet sind.

\section{Bendamustin und Anti-CD-20-Antikörper bei indolenten Lymphomen}

B endamustin wurde bisher in zwei Studien als M onotherapie eingesetzt [B remer et al., 1999; $\mathrm{H}$ eider et al., 1999]. D abei erzielte man bei vorbehandelten Patienten R emissionsraten von 67 und $80 \%$. E s ist vielfach beschrieben worden, dass Bendamustin auch nach vorheriger Alkylanzientherapie eingesetzt werden kann, da keine K reuzresistenz mit Cyclophosphamid besteht. In einer Studie zur Kombination von Chemotherapie und Rituximab fanden Czuczman und Kollegen [2000] 90\% ige R emissionsraten bei ausgewählten jüngeren Patienten mit follikulärem Lymphom.

$M$ athias Rummel, Frankfurt/M ., berichtete über ein Projekt der Studiengruppe für niedrig maligne Lymphome in Frankfurt: Entwickelt wurde ein Rezidivprotokoll als offene, prospektive, kontrollierte, multizentrische Phase-II-Pilotstudie mit R ituximab. D er A nti-CD -20-A ntikörper wird je nach Vorbehandlung der Patienten kombiniert mit Bendamustin oder $2 \mathrm{CdA} / \mathrm{M}$ itoxantron. D ie in Vortherapien eingesetzte Gesamtanthrazyklindosis wird berechnet, und bei Ü berschreiten der kardiotoxischen $\mathrm{G}$ renze wird stets $B$ endamustin statt $M$ itoxantron verwendet.

In die Studie eingeschlossen werden Patienten mit primärem Therapieversagen, mit frühem erstem Rezidiv (12 Monate) oder zweitem bzw. drittem Rezidiv der 4 indolenten Lymphome: Immunozytom, $M$ orbus Waldenström, $M$ antelzell-Lymphom und disseminiertes $M$ arginalzonen-L ymphom. Patienten mit langen R emissionzeiten (über 20 M onate) sind ausgeschlossen.

$\mathrm{E}$ in Z yklus B endamustin besteht aus $90 \mathrm{mg} / \mathrm{m}^{2}$ an 2 aufeinanderfolgenden Tagen pro Woche. R ituximab wird in der Dosierung von $375 \mathrm{mg} / \mathrm{m}^{2}$ insgesamt 6-mal gegeben. Zunächst wird 1 Woche vor Beginn der Chemotherapie Rituximab allein verabreicht. Die 2. Gabe erfolgt einen Tag vor Beginn des ersten B endamustin-Z yklus. D as R ituximab-B endamustinSchema mit 1-tägigem A bstand wird insgesamt 4-mal wiederholt. 4 Wochen danach erfolgt die $6 . \mathrm{G}$ abe von R ituximab.

B isher sind 15 Patienten im A Iter von median 62 Jahren in die Studien aufgenommen worden. Bei ihren E rkrankungen handelt es sich um 5 follikuläre Lymphome, $5 \mathrm{M}$. Waldenström, $4 \mathrm{M}$ antelzell-Lymphome und $1 \mathrm{M}$ arginalzonen-Lymphom. 10 Patienten hatten ein 1. Rezidiv, je 2 Patienten ein 2. bzw. 3. R ezidiv erlitten, und 1 Patient hatte nicht auf MCP- und CH OP-Therapien reagiert. E rste $\mathrm{H}$ inweise der bisher auswertbaren Patientendaten ergaben erstaunlich geringe $\mathrm{H}$ ämatotoxizität, 2-mal wurde eine kurzzeitige $\mathrm{G}$ ranulozytopenie $\mathrm{G}$ rad 3 beobachtet. E in stark vorbehandelter Patient hat eine partielle, 3 Patienten haben eine komplette R emission erreicht. E in Tumorlyse-Syndrom durch den A ntikörper wurde bisher nicht beobachtet.

B endamustin ist vergleichsweise nebenwirkungsarm und ein vielversprechender Kombinationspartner für den A nti-CD -20-A ntikörper R ituximab.

\section{Kombination von Bendamustin und Fludarabin bei rezidiviertem niedrig malignem NHL}

Fludarabin ergab als $M$ onotherapie in einer $M$ etaanalyse [Cheson, 1999], dass etwa 10\% von $326 \mathrm{~Pa}-$ tienten komplette Remission erreichten. Die Gesamtansprechrate lag bei etwa $50 \%$. Kombinationen von Fludarabin/M itoxantron/D examethason [M C Laughlin et al., 1996] und Fludarabin/R ituximab [Czuczman et al., 2000] bewirkten gute A nsprechraten und Remissionszeiten von bis zu 2 Jahren. Michael Herold, Erfurt, sieht daher in Fludarabin einen optimalen Kombinationspartner für B endamustin. $K$ eine der beiden Substanzen zeigt K reuzresistenzen zu Vortherapien mit A nthrazyklinen oder A lkylanzien.

In einer Phase-I/II-Studie an Patienten mit niedrig malignem NHL der Ostdeutschen Studiengruppe $\mathrm{H}$ ämatolgie/O nkologie (OSH O) wurden Fludarabin in einer Dosis von konstant $30 \mathrm{mg} / \mathrm{m}^{2}$ an den Tagen 1-3 und Bendamustin als Dosiseskalation, in den Dosierungsstufen 30,40 und $50 \mathrm{mg} / \mathrm{m}^{2}$ eingesetzt. Geplant sind maximal 6 Zyklen im A bstand von 4 Wochen. Kriterien der Studie sind maximal tolerierte Dosis, Freiheit von Therapieversagen, R emis- 
sionsrate sowie Feststellung der dosislimitierenden Toxizitäten.

Bisher wurden 15 vorbehandelte Patienten mit weit fortgeschrittener Erkrankung in die Studie aufgenommen. In der D osisstufe 1 ( $30 \mathrm{mg}$ Fludarabin und $30 \mathrm{mg}$ Bendamustin pro $\mathrm{m}^{2}$ ) gab es bei 9 Patienten zwei dosislimitierende Toxizitäten G rad 4, in Dosisstufe 2 (30 mg Fludarabin und $40 \mathrm{mg}$ Bendamustin pro $\mathrm{m}^{2}$ ) keine CTC-G rad-4-Toxizität bei 6 Patienten. In der letzten $\mathrm{G}$ ruppe musste jedoch die Therapie 2-mal über den Tag 35 hinaus verschoben werden. D ie A nsprechraten bei Dosislevel 1 ergaben 2 komplette R emissionen und 5 partielle R emissionen, von denen 3 eventuell als $\mathrm{CR}$ u (unconfirmed complete remission) bezeichnet werden können. 2 Patienten waren unter der Therapie progredient. Bei Dosislevel 2 erreichten bisher 3 von 4 Patienten partielle R emissionen.

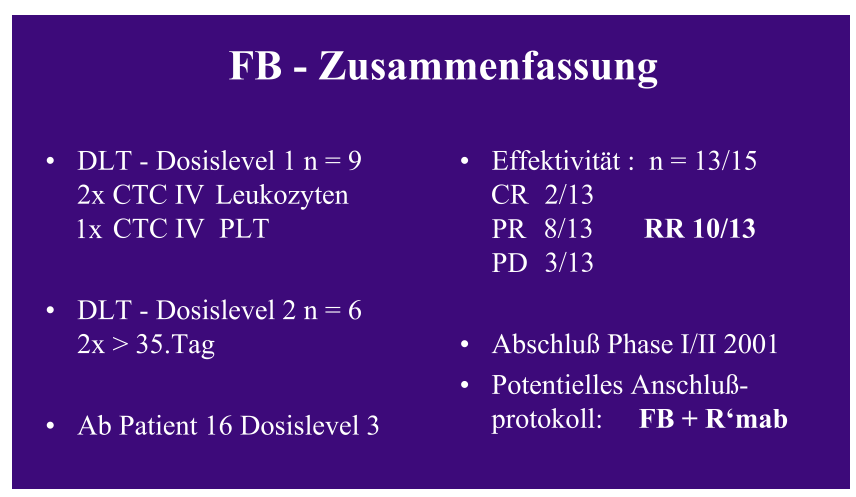

D ie Kombination von B endamustin und Fludarabin ist aufgrund fehlender $\mathrm{K}$ reuzresistenzen bei stark vorbehandelten Patienten mit rezidiviertem niedrig malignem N H L ein vielversprechendes Therapieregime.

\section{Bendamustin bei rezidivierten hoch malignen Lymphomen}

Gute L ebensqualität, Verträglichkeit, die M öglichkeit ambulanter B ehandlung und die in Kasuistiken beschriebene Wirksamkeit sprechen für eine Studie zu Bendamustin bei hoch malignen Lymphomen. Eckhardt Weidmann und Kollegen, Frankfurt/M., präsentierten die $\mathrm{D}$ aten einer PalliativtherapieStudie zu B endamustin bei rezidivierten hoch malignen und HIV-assoziierten Lymphomen. Eingeschlossen wurden 23 Patienten mit therapierefraktären Lymphomen, Frührezidiven, zweiten Rezidiven, Rezidiven nach $\mathrm{Hochdosistherapie} \mathrm{sowie}$ HIV-assoziierten Lymphomen, also Patienten, für die kurative Strategien nicht erfolgversprechend erscheinen.
Die meisten Patienten hatten extranodale $\mathrm{M}$ anifestationen und waren mit bis zu 4 verschiedenen Chemotherapien oder B estrahlung vorbehandelt, in 2 Fällen war autologe Stammzelltransplantation erfolgt.

B endamustin wurde in einer D osierung von $120 \mathrm{mg} / \mathrm{m}^{2}$ alle 3 Wochen verabreicht; bei lang anhaltend niedriger G ranulozytenzahl und Thrombopenie wurde die D osis auf $90 \mathrm{mg} / \mathrm{m}^{2}$ reduziert.

18 Patienten konnten bisher ausgewertet werden. D a sich bei HIV-Patienten die H elferzellen vermindern, spricht diese Patientengruppe auf die Therapie schlecht an. In einigen Fällen musste die Therapie aufgrund von progredienter $E$ rkrankung relativ früh wieder abgebrochen werden. B ei 4 Patienten wurde eine partielle, bei 4 weiteren eine komplette R emission erreicht, das bedeutet eine $\mathrm{G}$ esamtansprechrate von $44 \%$ bei diesem Patientenkollektiv mit schlechter Prognose. Die Remissionen waren überwiegend auf 2-3 M onate begrenzt, sie dauerten selten länger als $6 \mathrm{M}$ onate. 1 Patient befindet sich seit $12 \mathrm{M}$ onaten in R emission. B ei 2 Patienten musste die Therapie aufgrund von Toxizität G rad 3 bzw. G rad 4 abgebrochen werden. D ie nichthämatologischen Toxizitäten waren moderat.

Patienten mit fortgeschrittenen hoch malignen L ymphomen sprechen auf B endamustin an. A nsprechraten von $44 \%$ wurden erzielt. $D$ ie $L$ ebensqualität der Patienten war durch das Lymphom bestimmt und wurde durch B endamustin nicht eingeschränkt.

\section{Präklinik: Zytotoxizität von Bendamustin in resistenten Zellinien}

N orbert Schleucher, E ssen, berichtete über R esistenzund Interaktionsuntersuchungen an Tumorzelllinien mit Bendamustin, Paclitaxel, 5-FU und Vinorelbin. Die A rbeitsgruppe verwendete die Brustkrebszelllinen MCF-7 Wildtyp, deren A driamycin-resistente Variante und MDA-MB231. Weiterhin wurden die O varialkarzinom-Z elllinie A 2780, ihre A driamycinsowie Cisplatin-resistenten Subtypen und die humane Fibrosarkomzelllinie H T 1080 sowie der davon abgeleitete A driamycin-resistente Stamm untersucht.

A Is Z ytotoxizitätsassay setzten Schleucher und Kollegen einen modifizierten SR B (Sulforhodamin-B)-Test ein: eine bestimmte A nzahl Zellen wurde in 96-wellKulturplatten ausgesät. D ie Zellen wuchsen für $24 \mathrm{~h}$, danach erfolgte eine 2-stündige Inkubation mit den Zytostatika B endamustin, Paclitaxel oder V inorelbin bzw. eine 24-stündige Inkubation mit 5-Fluorouracil (5-FU ). N ach E ntfernung der Zytostatika inkubierten die Zellen bis zum Ende des Versuchs in Kulturme- 
dium. A nschließend wurden die Proteine mit Trichloressigsäure gefällt, mit SR B angefärbt und photometrisch gemessen. $D$ ie Proteinkonzentration diente als $\mathrm{M}$ aß für das Wachstum der Zellen.

$M$ an untersuchte zunächst die zytotoxische $A$ ktivität des Bendamustins in A driamycin-resistenten Zelllinien, verglichen mit der entsprechenden Wildtyplinie. $D$ ie resistenztragenden Varianten von M CF-7 und A 2780 zeigen den M D R-1(multi drug resistance 1)Phänotyp, exprimieren also das p170-G lykoprotein in großer $M$ enge. $D$ abei handelt es sich um eine Pumpe zum A usschleusen niedermolekularer, toxischer Substanzen aus der Zelle. Tumorzellen gewinnen durch das Protein einen Selektionsvorteil: Es führt zu beschleunigtem E fflux von Zytostatika.

Die Zelllinien mit MDR-1-Phänotyp werden durch B endamustin gleichermaßen gehemmt wie die entsprechenden Wildtyp-Zelllinien. MDR führte in diesen Experimenten also nicht zu Bendamustin-R esistenz. Für die $\mathrm{H}$ emmung der $\mathrm{A}$ driamycin-resistenten HT1080-Zelllinie benötigt man jedoch eine 5-fach höhere Bendamustin-Konzentration als beim Wildtyp, um die IC50-Konzentration zu erreichen. Der R esistenzfaktor dieser Z elllinie ist nicht MDR, sondern das 190-kD a-M ultiresistenzprotein. D ie Cisplatin-resistente $O$ varialkarzinomzelllinie benötigt ebenfalls höhere Bendamustin-Konzentrationen zum E rreichen der IC50-Konzentration als der Wildtyp. Der R esistenzmechanismus dieser Zelllinie ist noch nicht abschließend untersucht.

A us diesen U ntersuchungen kann man schließen, dass MDR-Zellinien durch Bendamustin gehemmt werden. Die Wirkung erfolgt sehr schnell; längere Inkubationszeiten als 2 h brachten keinen zusätzlichen Effekt.

\section{Bendamustin-Interaktionen mit Vinorelbin und Paclitaxel}

Schleucher untersuchte mit $\mathrm{H}$ ilfe des Zytotoxizitätsassays auch die Interaktionen der Zytostatika B endamustin, Vinorelbin, 5-FU und Paclitaxel, wobei zusätzlich überprüft wurde, ob die R eihenfolge der Substanzen einen Einfluss auf ihre Wirkung hat. $M$ ittels Isobologramm nach Behrenbaum wurden Synergismus, A ntagonismus bzw. additives Verhalten der Zytostatika in verschiedenen Zelllinien ermittelt. $\mathrm{E}$ in interessantes E rgebnis dieser Experimente war der Synergismus von B endamustin und $V$ inorelbin in beiden B rustkrebszelllinien unabhängig von der R eihenfolge, in der die Substanzen eingesetzt worden waren. Diese Experimente könnten die Grundlage für ein vielversprechendes Studiendesign werden.
Interaktionen der Zytostatika B endamustin, Paclitaxel und Vinorelbin in den B rustkrebszelllinen M CF-7 (Wildtyp) und M DA -M B 231 ${ }^{\text {a }}$

\begin{tabular}{|c|c|c|}
\hline MCF-7 & $\begin{array}{l}\text { B endamustin vor 5-FU: } \\
\text { 5-FU vor Bendamustin: }\end{array}$ & $\begin{array}{l}\text { synergistisch } \\
\text { additiv }\end{array}$ \\
\hline M DA -M B 231 & $\begin{array}{l}\text { Bendamustin vor 5-FU: } \\
\text { 5-FU vor Bendamustin: }\end{array}$ & $\begin{array}{l}\text { synergistisch } \\
\text { antagonistisch }\end{array}$ \\
\hline MCF-7 & $\begin{array}{l}\text { B endamustin vor Paclitaxel: } \\
\text { Paclitaxel vor B endamustin: }\end{array}$ & $\begin{array}{l}\text { synergistisch } \\
\text { antagonistisch }\end{array}$ \\
\hline M B 231 & $\begin{array}{l}\text { B endamustin vor Paclitaxel: } \\
\text { Paclitaxel vor B endamustin: }\end{array}$ & $\begin{array}{l}\text { antagonistisch } \\
\text { synergistisch }\end{array}$ \\
\hline MCF-7 & $\begin{array}{l}\text { B endamustin vor Vinorelbin: } \\
\text { Vinorelbin vor Bendamustin: }\end{array}$ & $\begin{array}{l}\text { synergistisch } \\
\text { synergistisch }\end{array}$ \\
\hline M DA -M B 231 & $\begin{array}{l}\text { B endamustin vor V inorelbin: } \\
\text { Vinorelbin vor B endamustin: }\end{array}$ & $\begin{array}{l}\text { synergistisch } \\
\text { synergistisch }\end{array}$ \\
\hline
\end{tabular}

a Inkubationen mit B endamustin, Paclitaxel und Vinorelbin für $2 \mathrm{~h}$, mit 5-FU für $24 \mathrm{~h}$.

B endamustin zeigte signifikante Zytotoxizität in humanen Tumorzellinien, auch jenen mit M D R-1A ktivität. D ie Sequenz B endamustin und 5-FU offenbarte additive bis synergistische I nteraktionen. $B$ endamustin und Vinorelbin ergaben einen sequenzunabhängigen Synergismus in zwei verschiedenen Z elllinien. D ie zytotoxische A ktivität von Paclitaxel wird möglicherweise durch B endamustin erhöht.

\section{Apoptose auf neuen Wegen?}

Synergismus und A ntagonismus von B endamustin in Kombination mit anderen Medikamenten war auch Thema des Vortrags von Kai Chow, Frankfurt/M . In seinen Experimenten waren die Kombinationspartner von Bendamustin: Doxorubicin, M itoxantron, Cladribin oder der A nti-CD-20-A ntikörper R ituximab. Die A rbeitsgruppe arbeitete sowohl mit Zelllinien, den follikulären Lymphomlinien $\mathrm{D} \mathrm{OH} \mathrm{H-2} \mathrm{und}$ WSU-NHL, als auch mit Zellen, die von Patienten mit $C L L$, leukämischen niedrig malignen $\mathrm{B}-\mathrm{NHL}$ oder T-N HL stammten. D ie R eaktion der Zellen auf Bendamustin und auf die Kombinationspartner wurde mit 5 verschiedenen A poptose- und Proliferationsassays untersucht. Die Effektorproteine der A poptose und p53 wurden mit spezifischen A ntikörpern auf Western-B lots nachgewiesen.

Bendamustin und Doxorubicin bzw. Mitoxantron zeigten dosisabhängig strengen A ntagonismus, während für die Kombination mit Cladribin additive $\mathrm{E}$ ffekte festgestellt wurden. $\mathrm{R}$ ituximab in Kombination mit $B$ endamustin reduzierte die IC50-Konzentration von Bendamustin und verstärkte die A poptose, verglichen mit B endamustin allein. 
B ei eingehender $A$ nalyse der A poptose-K askade und der A ktivierung desTumorsuppressors $p 53$ stellte sich heraus, dass die Bendamustin-vermittelte A poptose offenbar von p53 unabhängig ist. A uch $E$ ffektoren der A poptose, z. B. B Cl-2-Proteine, Caspasen und PA R P, werden durch Bendamustin nicht beeinflusst. Wird Bendamustin zusammen mit anderen Zytostatika verwendet, so bestimmt der Kombinationspartner, nicht B endamustin, die A ktivierung, Expression bzw. Spaltung der an A poptose beteiligten Proteine. Da Bendamustin in A ssay-Systemen, die apoptotische Zellen nachweisen, wirksam ist, p53 und bekannte E ffektoren der A poptose aber nicht beeinflusst werden, muss man einen weiteren, bisher unbekannten A poptoseweg postulieren. Möglicherweise kommen die antagonistischen Wirkungen von Bendamustin und $D$ oxorubicin bzw. Mitoxantron durch gegenseitige Hemmung des bekannten und unbekannten A poptosewegs zustande.

B endamustin induziert die A poptose in Lymphomzellen und Z elllinien. Die E xperimente führen möglicherweise zu neuen E rkenntnissen über A poptose.

\section{Bendamustin in der Radioonkologie}

$\mathrm{H}$ ans-Peter Rodemann, Tübingen, untersuchte mit seinen Kollegen Kulturen menschlicher Zellinien nach Bestrahlung bzw. Bestrahlung in Kombination mit B endamustin. B estrahlung von Z ellen führt entweder direkt oder durch Bildung von Sauerstoffradikalen zu DNA -Schäden. Weiterhin werden p53 und zahlreiche weitere Signalwege aktiviert, die entweder R eparatur oder A poptose einleiten. Fibroblasten des Bindegewebes aktivieren hingegen nach B estrahlung überwiegend $D$ ifferenzierungsprozesse, die beim $M$ enschen in $\mathrm{H}$ aut und Lunge zu Fibrosen führen können.

R odemann erwartet von seinen Experimenten $\mathrm{H}$ inweise auf synergistische $E$ ffekte durch Strahlung und B endamustin, die für eine zukünftige Strahlenchemotherapie bedeutsam sein könnten.

$D$ ie untersuchten Zelllinien sind die Mammakarzinomzelllinie M DA -M B 231 mit mutierten p53-G enen und die Bronchialkarzinomzelllinie A 549 mit Wildtyp-p53; als Kontrollen dienten die $\mathrm{H}$ autfibroblastenlinie H SF 6 oder die embryonalen L ungenfibroblasten CD 1032. Die Bendamustin-K onzentrationen in den Experimenten variierten zwischen 10 und $250 \mathrm{~mm}$, was ungefähr $50-100 \mathrm{mg} / \mathrm{m}^{2}$ in der Therapiesituation in vivo entspricht. 4-mal pro Woche wurden die Kulturen behandelt; entweder wurde nur bestrahlt, oder vor der Bestrahlung wurde Bendamustin zugegeben. $8 \mathrm{~h}$ danach wurden Koloniebildungstests ausgesät. In der Z wischenzeit hatten die Zellen Gelegenheit, die D NA -Schäden zu reparieren.

Die klonogene A ktivität der Z elllinien nach B endamustin-B ehandlung und B estrahlung war sehr unterschiedlich. Im Vergleich zu Bestrahlung allein sah Rodemann bei einer Konzentration von $50 \mathrm{~mm}$ Bendamustin und nachfolgender B estrahlung eine synergistische Hemmung der Brustkrebszellinie MDA M B 231. B ei der L ungenkrebszelllinie A 549 konnte hingegen auch bei höheren D osen B endamustin kein synergistischer Effekt mit Bestrahlung beobachtet werden. Stattdessen wurden bei dieser Z elllinie interessante R esistenzphänomene beobachtet, deren $U$ rsache noch genauer untersucht werden. A uch die beiden Fibroblastenlinien reagierten auf die Kombinationsbehandlung kaum empfindlicher als auf ausschließliche B estrahlung.

E s gibt einen synergistischen $\mathrm{H}$ emmeffekt von

B estrahlung und $B$ endamustin, der aber möglicherweise nur bei bestimmten Zelllinien bzw. Tumor-E ntitäten zur Wirkung kommt.

\section{Apoptose bei CLL}

Ziel jeder zytotoxischen Therapie ist die A poptose der Tumorzellen. Folke Schriever, Wartenberg, und seine Kollegen an der Charité, B erlin, untersuchten die A poptose von CL L-Zellen, die sie aus peripherem Blut von Patienten isoliert hatten. Die A poptose der $C L L-Z$ ellen ist offensichtlich gehemmt. U rsächlich, aber vermutlich nicht allein verantwortlich dafür ist die Ü berexpression des A poptose-hemmenden bcl-2Proteins. D ie Zellen sind aufgrund ihres I mmunstatus (CD 5-, CD 19-, CD 20-, CD 23- und CD 38-Expression) leicht zu isolieren.

Schriever interessierte hauptsächlich, ob es möglich ist, die Sensitivität von CLL-Zellen gegenüber Bendamustin nachzuweisen und ob in-vitro-Daten bei der Auswahl von Patienten, die von Bendamustin profitieren würden, helfen können.

$M$ it dem A nnexin-5-A poptose-A ssay und FACSA nalytik wurden zunächst $D$ osis und E inwirkungszeit bis zur A poptose bestimmt. $\mathrm{Zu}$ maximaler A poptose-Induktion kam es bei $0,125 \mathrm{mM}$ Bendamustin nach $48 \mathrm{~h}$. E ine weitere Steigerung der A poptose- $R$ ate durch höhere Konzentrationen von Bendamustin war nicht möglich. $40-50 \%$ aller CLLZellen lassen sich also auch bei Steigerung der Bendamustin-Konzentration nicht in die A poptose treiben. Dafür sind primäre oder sekundäre Resistenzmechanismen verantwortlich. Es stellt sich die Frage, ob andere Zytostatika die A poptose der CLL Zellen verstärken können. D azu wurde B endamustin 
$(0,125 \mathrm{mM})$ mit Dexamethason (100 ng/ml) oder Fludarabin $(500 \mathrm{ng} / \mathrm{ml})$ kombiniert.

Bendamustin hat mit Dexamethason und Fludarabin keinen synergistischen Effekt

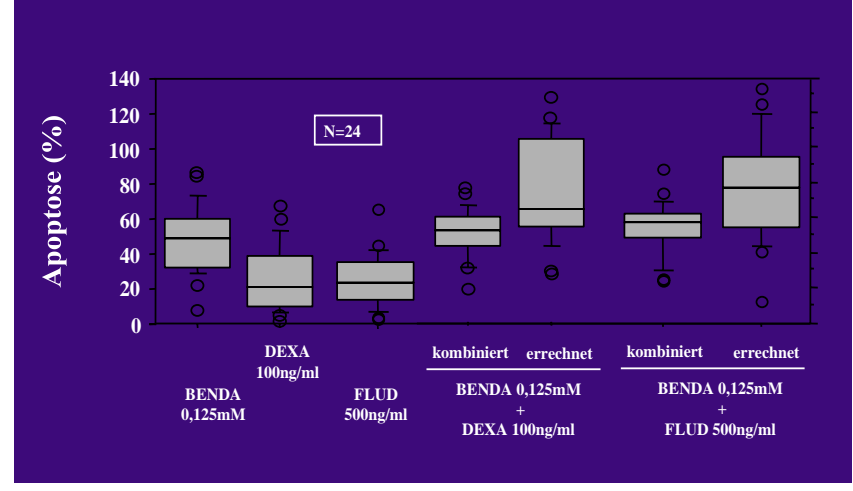

In den Experimenten konnte kein synergistischer oder additiver $\mathrm{E}$ ffekt durch die Kombinationspartner nachgewiesen werden. $D$ examethason allein bewirkte $20 \%$, Fludarabin allein 24\% A poptose.

Korrelationen der in-vitro-D aten mit dem klinischen Verlauf der Krankheit des Patienten, von dem die CLL-Zellen stammen, wurden nicht gefunden.

In-vitro-Effekt von Bendamustin korreliert nicht mit klinischen Parametern

- Leukozyten

$-\mathrm{Hb}$

- Thrombozyten

- Stadium der B-CLL (Binet)

- Geschlecht

- Zeitraum bis Progress

- Vorbehandlung der B-CLL

- Anzahl und Art der Vortherapie

$D$ ie A poptose-Induktion durch B endamustin ist zeitund dosisabhängig. E s gibt in vitro keinen synergistischen oder additiven $E$ ffekt von B endamustin in Kombination mit $D$ examethason oder Fludarabin. $D$ ie experimentellen $D$ aten stehen in keinem offensichtlichen Z usammenhang mit klinischen $D$ aten des Patienten, von dem die auf B endamustin-vermittelte A poptose untersuchten CL L -Zellen stammen. O ffenbar verfügt ein Teil der $C L L$-Z ellen primär über einen R esistenzmechanismus gegen $B$ endamustin-induzierte A poptose. D ieser molekulare M echanismus sollte weiter untersucht werden.

\section{Stammzell- und Immunotoxizität}

K laus Ruffert, Jena, untersuchte die Wirkung von Bendamustin in Kombination mit Etoposid auf Stammzellen und I mmunsystem.

7 Patienten mit CLL und 7 mit anderen NH L wurden alle 3 Wochen mit B endamustin behandelt: $100 \mathrm{mg} / \mathrm{m}^{2}$ an Tag 1, zusätzlich E toposid: oral $50 \mathrm{mg}$ absolut an den Tagen 1-5. Im M ittel erhielten die Patienten 7,7 Zyklen. Der Immunstatus wurde vor der Therapie, nach 6 Zyklen und 6 Monate nach A bschluss der B ehandlung untersucht.

$D$ as $T 4 / T$ 8-Verhältnis lieferte bereits in früheren U ntersuchungen R ufferts keine klare A ussage über die Zytotoxizität einer Therapie. A us diesem G rund wurden $\mathrm{K}$ nochenmarkuntersuchungen mittels $\mathrm{D}$ urchflusszytometrie (FACS) zum Nachweis CD 34-positiver Zellen durchgeführt. D ie A nzahl von Vorläuferzellen der Granulozyten und Monozyten und ihr Wachstum wurden im Kultur-A ssay als CFU (colony forming units) bestimmt.

Zur Beurteilung der zellulären Immunität im Blut dienten FA CS-U ntersuchungen der beiden Subpopulationen von $\mathrm{T}$-H elferzellen. $\mathrm{D}$ ie $\mathrm{H}$ elferfunktion bei der $\mathrm{MHC}$-abhängigen Differenzierung von $\mathrm{B}$ - und Killerzellen wurde anhand des intrazellulären Interferons $\gamma$ der $\mathrm{TH}_{1}$-Zellen bestimmt, die M HC-unabhängige $\mathrm{A}$ ktivierung ruhender $\mathrm{B}$-Zellen über den $\mathrm{N}$ achweis von II-4 in $\mathrm{TH}_{2}$-Zellen.

Die Patienten mit NHL hatten vor der Therapie im Knochenmark durchschnittlich 0,36\% CD-34-positive Zellen, danach erhöhte sich der A nteil auf 0,43\%. E in halbes Jahr später war der Prozentsatz wieder etwas abgefallen.

$D$ ie CL L -Patienten hatten vor der Therapie geringere A usgangswerte. Danach stieg der Stammzellanteil auf das D oppelte an und war $6 \mathrm{M}$ onate später noch konstant.

In den Kultur-A ssays mit Vorläuferzellen der Granulozyten und Monozyten sah Ruffert Stimulation bei NHL- und CLL-Patienten nach der Therapie. $6 \mathrm{M}$ onate nach $\mathrm{A}$ bschluss der Behandlung verringerten sich die CFU wieder, blieben aber höher als vor der Therapie.

Sowohl N HL - als auch CL L-Patienten wiesen vor der Therapie niedrige $\mathrm{TH}_{1}$ - und $\mathrm{TH}_{2}$-Werte auf. $\mathrm{N}$ ach der Therapie verdoppelten sich Interferon- $\gamma$ - bzW. II-4Werte dieser Zellpopulationen und blieben $6 \mathrm{M}$ onate lang auf diesem $\mathrm{L}$ evel. $\mathrm{D}$ ie B estimmung der $\mathrm{TH}_{1}$ - und $\mathrm{TH}_{2}$-A ktivitäten hat die Beurteilung dieser Wirkungen erleichtert.

$D$ ie Therapie mit B endamustin/E toposid bewirkt bei CL L - und anderen N H L -Patienten eine N ormalisierung der I mmunantwort für mindestens ein halbes J ahr. D ie 
L eukozytenproliferation normalisiert sich durch die

Therapie. D ie Z unahme der Stammzellreserve im

$K$ nochenmark ist bis zu einem halben Jahr nach

Therapieabschluss nachweisbar. B endamustin in Kom-

bination mit $E$ toposid zeigt in der verwendeten

D osierung keine Stammzell toxizität.

\section{Bendamustin als «Salvage»-Therapie beim Mammakarzinom}

Ursula Reichmann, Tübingen, berichtete von $D$ aten einer Palliativ-Studie mit 20 auswertbaren Patientinnen mit gesichertem Mammakarzinom, Fernmetastasen und nachgewiesener Tumorprogression. $\mathrm{D}$ ie Patientinnen hatten vor Studienbeginn bis zu 7 Chemotherapien (ohne B endamustin) erhalten.

$D$ ie D osis von B endamustin betrug $120 \mathrm{mg} / \mathrm{m}^{2}$, intravenös, über $30 \mathrm{~min}$, an den Tagen 1 und 2. Dieses Schema wurde am 29. Tag wiederholt, wenn A llgemeinbefinden und Laborparameter dies erlaubten. $D$ as Durchschnittsalter der Patientinnen betrug 58 Jahre. M edian erhielten sie 4 Z yklen.

D ie Therapie war bei vielen Patientinnen von Ü belkeit begleitet. Erbrechen war eine häufige unerwünschte Wirkung. Da dieser toxische $E$ ffekt unter Bendamustin eher selten in schwerem A usmaß vorkommt, wurde «antizipatorisches Erbrechen» als mögliche U rsache diskutiert; die Patientinnen hatten offenbar zu negative Erfahrungen mit den vorausgegangenen Chemotherapien gemacht. Zur B ehandlung wurden Serotonin-A ntagonisten, D examethason, gelegentlich sogar Sedativa eingesetzt. Weiterhin kam es bei $20 \%$ der Patientinnen zu Leukopenien $\mathrm{G}$ rad 3. Schwerwiegende I nfektionen traten nicht auf. E ine G rad-4-Thrombopenie war limitierend für die B ehandlung. Nur in einem Fall wurde milde A lopezie festgestellt, 1-mal eine allergische $R$ eaktion.

3 Patientinnen werden derzeit noch innerhalb der Studie behandelt, ihre Erkrankungen sind bisher stabil. Es kam zu keiner kompletten Remission, in $20 \%$ der Fälle wurde partielle R emission festgestellt, in $50 \%$ stabile E rkrankung. B ei $25 \%$ war der Tumor primär progredient. Das progressionsfreie Intervall dauerte median 4 Monate, das längste dauerte $9 \mathrm{M}$ onate.

In einer vergleichbaren, aber multizentrischen Studie, die von $\mathrm{K}$ laus $\mathrm{H}$ öffken, J ena, geleitet wurde, erreichten 22\% der Patientinnen partielle Remission, 3\% komplette R emission und $56 \%$ stabile Erkrankung. In $17 \%$ der Fälle kam es zu M yelosuppression vom Schweregrad 3-4.

B endamustin in einer D osierung von $120 \mathrm{mg} / \mathrm{m}^{2}$ ist wirksam bei Patientinnen mit mehrfach rezidiviertem und vorbehandeltem M ammakarzinom. DieWirksamkeit ist unabhängig von der A nzahl der Vorbehandlungen und einer Vortherapie mit Taxanen oder A nthrazyklinen. E s gibt wenig dosislimitierende Toxizitäten; Ü belkeit und $E$ rbrechen erfordern bei stark vorbehandelten Patientinnen häufig A ntiemese.

\section{Bendamustin bei kleinzelligem Bronchial- karzinom (SCLC)}

M an unterscheidet beim SCLC zwei Stadien: «limited» und «extensive disease». B ei begrenzter E rkrankung strebt man eine kurative Therapie an, obwohl nur $10-20 \%$ der Patienten in diesem Stadium 5 Jahre überleben. Bei ausgedehnter E rkrankung ist die Therapie palliativ, die mittlere Ü berlebenszeit beträgt 8-10 M onate, nach 2 Jahren leben noch $10 \%$ der Betroffenen. Langzeitüberleben findet man bei diesem Tumor extrem selten.

B ei «extensive disease» ist die Verwendung einer neuen Substanz in der Primärtherapie ethisch vertretbar. N orbert $\mathrm{N}$ iederle, L everkusen, berichtete von einer Studie an 32 Patienten mit SCLC im fortgeschrittenen Stadium, die weder mit Strahlen- noch mit Chemotherapie vorbehandelt waren. Sie erhielten $120 \mathrm{mg} / \mathrm{m}^{2}$ B endamustin an den Tagen 1 und 2 als 1-stündige Infusion. Nach 3 Wochen wurde dieses $\mathrm{R}$ egime wiederholt. $\mathrm{D}$ ie Patienten erhielten median 3 Zyklen. $\mathrm{H}$ insichtlich der A nsprechraten konnten 24 Patienten beurteilt werden, hinsichtlich Toxizität 28.

Die R emissionsrate betrug 50\% , 3 Patienten erreichten eine komplette, 9 eine partielle Remission, 4 hatten eine stabile Erkrankung. E in Drittel der Tumoren war primär progredient. Die Remission dauerte im Median 3 (Range 1-12) Monate. Die Ü berlebenszeiten waren median 8 (2-12) M onate. Die hauptsächlichen Nebenwirkungen waren Ü belkeit und Erbrechen. Zu Grad 3 bis 4-Toxizitäten kam es nur bei 2 Patienten: L eukozytopenie G rad 3 und A nämie bzw. Thrombozytopenie Grad 3. Zwei Patienten erlitten allergische Reaktionen, die zum A bbruch der Therapie führten. A lopezie trat nicht auf.

Wolf $K$ öster, E ssen, hat den $K$ rankheitsverlauf von 18 SCLC-Patienten, die eine Palliativtherapie mit $B$ endamustin erhalten hatten, retrospektiv analysiert. A lle Patienten waren intensiv vorbehandelt; die meisten hatten $R$ ezidive; 4 Patienten erhielten B endamustin, nachdem eine $E$ rstlinien-Therapie mit anderen Zytostatika aufgrund von Toxizität abgebrochen werden musste.

D ie Therapie mit B endamustin ergab, außer im hämatologischen B ereich, keine W H O-G rad 3 oder 4-Toxi- 
zitäten. Bei den Patienten, die Bendamustin als Z weitlinien-Therapie bekommen hatten, war die $\mathrm{H}$ ämatotoxiziät höher als bei jenen, die es als E rstlinien-Therapie erhalten hatten.

$\mathrm{Zu}$ partieller Remission kam es bei $32 \%$, bei der $\mathrm{H}$ älfte der Patienten stabilisierte sich die $\mathrm{K}$ rankheit, bei $20 \%$ war der Tumor progredient.

Patienten mit besserem A llgemeinbefinden profitierten eher von der B endamustin-Therapie als Patienten mit niedrigem $\mathrm{K}$ arnofsky-Index. Dies spiegelte sich sowohl im rezidivfreien Überleben wie auch im $\mathrm{G}$ esamtüberleben wider. Jedoch fand sich auch bei Patienten in $Z$ weitlinien-Therapie nur geringe nichthämatologische Toxizität, die $\mathrm{Hämatotoxizität} \mathrm{war}$ moderat.

A ufgrund der guten $D$ atenlage starteten $K$ öster und Kollegen im Februar dieses Jahres eine Studie an nicht vorbehandelten SCLC-Patienten im Stadium «extensive disease». A Is Phase-I/II-Protokoll wird eine Kombination von B endamustin und $C$ arboplatin geprüft.

B endamustin zeigt deutlicheVorteile in der B ehandlung von Patienten mit SCL C im Stadium «extensive disease».

$D$ ie medianen Remissions- und Ü berlebenszeiten sind denen bei Standardtherapien vergleichbar, wobei die

$\mathrm{N}$ ebenwirkungen deutlich geringer sind.

B endamustin ist eine vielversprechende therapeutische

A Iternative bei Patienten mit SCL C im Stadium «extensive disease». B esonders ältere Patienten oder solche mit schlechtem A llgemeinzustand können davon profitieren.

\section{Radiochemotherapie von Kopf-Hals- Tumoren}

Fortgeschrittene Plattenepithelkarzinome im Kopf$\mathrm{H}$ als-B ereich werden in der $\mathrm{R}$ egel multimodal behandelt. A Is Primärtherapie erfolgt zunächst eine radikale O peration, dann R adiochemotherapie. Z usätzlich gibt es K onzepte für präoperatives «downstaging» mit $\mathrm{R}$ adio- oder systemischer $\mathrm{C}$ hemotherapie. Viele $\mathrm{Pa}$ tienten können jedoch nicht kurativ behandelt werden. D ie R ezidivrate ist hoch, so dass im fortgeschrittenen Statium häufig nur noch palliative Therapieoptionen bestehen, auch weil wegen hoher Komorbidität eine $N$ arkose nicht mehr möglich ist.

A ngelika Rahn, Frankfurt/M., berichtete über eine Studie mit palliativem Therapiekonzept. 15 vorbehandelte Patienten mit 1-4 R ezidiven im A Iter von median 56 Jahren und einem Karnofsky-Index bis $70 \%$ erhielten eine $\mathrm{R}$ adiochemotherapie mit Bendamustin. $D$ ie $R$ adiotherapie bestand meistens in einer Photonenenbestrahlung, klassischen 3D-B estrah- lungsserien mit isozentrischen Stehfeldern, Maskenfixierung und Lamellenkoordination zur Schonung des Rückenmarks. Nur der Tumor selbst wurde bestrahlt. D ie D osis war $3 \mathrm{G}$ y täglich, an 5 aufeinanderfolgenden Tagen. $A n$ den Tagen 1 und 2 erhielten die Patienten zusätzlich Bendamustin in der $\mathrm{Maxi-}$ maldosis von $150 \mathrm{mg} / \mathrm{m}^{2}$, dazu eine antiemetische Therapie. Die Toxizität war sehr mild. 10 Patienten gaben keine subjektiven Nebenwirkungen an, ansonsten traten überwiegend Toxizitäten der $\mathrm{G}$ rade 1 oder 2 auf. Grad 3 oder 4-Toxizitäten erlitten 2 Patienten (Fieber, Verwirrtheit, E rbrechen bzw. E rbrechen und Tumorulkus). Die A nsprechrate lag bei 50\%, 4 partielle und 4 komplette Remissionen wurden dokumentiert. 2 Patienten zeigten Symptomminderung ohne Verkleinerung des Tumors. 1 Patient sprach auf die Therapie nicht an. Die Remissionsdauer der 10 Patienten, für die ein Follow-up möglich war, betrug im M ittel 19 Wochen.

Die Ü berlebenszeit war median 29 Wochen. Bei einem Patienten betrug sie jedoch 2 Jahre. Rahn schilderte das Fallbeispiel eines Patienten, der 10 Jahre nach primärer $\mathrm{R}$ adiochemotherapie eines K eilbeinhöhlenkarzinoms ein Rezidiv erlitt. Er wurde im A ugust 1997 bestrahlt und erlebte eine gute partielle R emission über fast $1 \mathrm{~J}$ ahr. E rst $13 \mathrm{M}$ onate nach der Palliativtherapie wurde er progredient und ist nach einem weiteren Jahr verstorben.

D as palliative Radiochemotherapieschema mit $B$ endamustin ist für intensiv vorbehandelte $P$ atienten mit Kopf-H als-Tumoren gut verträglich. D ie M ehrzahl der Patienten litt nicht unter theapiebedingten unerwünschten Wirkungen. D ieA nsprechraten sind gut und entsprechen jenen wesentlich toxischerer Therapien.

\section{Literatur}

A nger G, Fink R, Fleischer J, H esse P, K rug K, R aderecht C, R ieche K, Subert L, Wutke $K$ : Vergleichsuntersuchungen zwischen Cytostasan und Cyclophosphamid bei der chronischen Lymphadenose, dem Plasmozytom, der Lymphogranulomatose und dem Bronchialkarzinom. D tsch G esundheitswesen 1975; 30:1280-1285.

B remer $\mathrm{K}$ : B endamustin: $\mathrm{E}$ ffective salvage chemotherapy in pretreated low grade Non-H odgkin-Lymphomas (L.G r.NHL). A nn O ncol 1999;10(suppl 3): 127.

Cheson BD: N ew chemotherapeutics strategies for the treatment of indolent lymphoid malignancies. R eview. Semin Hematol 1999;36(suppl 5):26-33.

Czuczman MS, Jonas C, Stephan M, Scarpace A, Rock M, Kunkel L, G rillo-L opez A . B ernstein SH : Pilot study of rituxan in combination with fludarabine chemotherapy in patients with low-grade or follicular non-H odgkin's lymphoma. Blood 2000;96:729a (abstr 3154).

Dohner $H$, Stilgenbauer $S, B$ enner $A$, Leupolt $E, K$ rober $A, B$ ullinger $L$, D ohner K, B entz M, Lichter P: G enomic aberrations and survival in chronic lymphocytic leukemia. N E ngl J M ed 2000;343:1910-1916. 
D ohner H, Fischer K, B entz M, H ansen K, Benner A, Cabot G, D iehl D, Schlenk $R$, Coy J, Stilgenbauer S: p53 gene deletion predicts for poor survival and non-response to therapy with purine analogs in chronic B-cell leukemias. Blood 995; 85: 1580-1589.

H amblin TJ, D avis Z, G ardiner A , O scier D G, Stevenson FK : U nmutated Ig V (H) genes are associated with a more aggressive form of chronic lymphocytic leukemia. Blood 1999;94:1848-1854.

H eider A , K öster W, G rote-K iehn J, B remer K, Wilke H, N iederle N : B endamustin in untreated small cell lung cancer (SCLC): E fficacy and toxicity. E ur J Cancer 1999;35(suppl 4):254.

\section{Referenten}

D r. M anuel A ivado, U niversitätsklinikum D üsseldorf

D r. K ai Chow, K linikum der J.W. G oethe-U niversität, Frankfurt/M .

D r. M artin D reyling, L udwig-M aximilians- $U$ niversität $M$ ünchen

Prof. D r. B ertold $E$ mmerich, $L$ udwig-M aximilians- $U$ niversität $M$ ünchen

D r. M ichael Herold, K linikum E rfurt G mbH, Erfurt

Prof. D r. K laus H öffken, K linikum der Friedrich-Schiller U niversität, Jena

D r. Wolf K öster, K liniken E ssen-M itte, E ssen

Prof. D r. Paris S. M itrou, K linikum der J.-W.-G oethe-U niversität, Frankfurt/M.

Prof. D r. Norbert N iederle, K linikum L everkusen

D r. A ngelika R ahn, K linikum der J.-W.-G oethe-U niversität, Frankfurt/M.

D r. U rsula R eichmann, U niversitätsklinikum Tübingen

D r. H ans-Peter R odemann, U niversitätsklinikum Tübingen

PD D r. K laus R uffert, O nkologische Schwerpunktpraxis, Jena

D r. M athias R ummel, K linikum der J.-W.-G oethe-U niversität, Frankfurt/M.

D r. N orbert Schleucher, U niversitätsklinikum E ssen

PD Dr. Folke Schriever, K linik Wartenberg

PD D r. Wolfgang Schultze, HU M A INE K linikum, Bad Saarow

D r. E ckhardt Weidmann, K linikum der J.-W.-G oethe-U niversität, Frankfurt/M
$\mathrm{K}$ ath $\mathrm{R}$, B lumenstengel $\mathrm{K}$, Fricke $\mathrm{HJ}, \mathrm{H}$ offken $\mathrm{K}$ : B endamustine monotherapy in advanced and refractory chronic lymphocytic leukemia. J Cancer R es Clin O ncol 2001;127:48-54.

M CL aughlin P, Seymour J, Fuller L, Cabanillas F: Combined modality therapy for stage I-II MALT lymphoma and mantle cell lymphoma. A nn Oncol 1996;7: 211-213.

M olica S: Progression and survival studies in early chronic lymphocytic leukemia. Blood 1991;78:895-899.

\section{Impressum}

\section{B eilage zu \\ O nkologie, B and 24, H eft 3, J uni 2001}

A utorin: D r. M aren M undt, Freiburg i. Br.

$M$ it freundlicher $U$ nterstützung durch ribosepharm $\mathrm{G} \mathrm{mbH}$, $M$ ünchen

D ie Wiedergabe von G ebrauchsnamen, $\mathrm{H}$ andelsnamen, Warenbezeichnungen etc. in dieser Beilage auch ohne besondere Kennzeichnung berechtigt nicht zu der A nnahme, dass solche Namen im Sinne der Warenzeichen- und $M$ arkenschutz-G esetzgebung als frei zu betrachten wären und daher von jedermann benutzt werden dürften. Für A ngaben über D osierungsanweisungen und A pplikationsformen kann vom Verlag keine Gewähr übernommen werden. Derartige A ngaben müssen vom jeweiligen A nwender im E inzelfall anhand anderer L iteraturstellen auf ihre R ichtigkeit überprüft werden.

A lle R echte vorbehalten.

Ohne schriftliche Genehmigung des Verlags dürfen diese Publikation oder Teile daraus nicht in andere Sprachen übersetzt oder in irgendeiner Form mit mechanischen oder elektronischen Mitteln (einschließlich Fotokopie, Tonaufnahme und M ikrokopie) reproduziert oder auf einem $\mathrm{D}$ atenträger oder Computersystem gespeichert werden.

(C) Copyright 2001 by S. K arger $\mathrm{G} \mathrm{mbH}$, Postfach,

D -79095 Freiburg, und S. K arger A G, Postfach, CH -4009 B ase D ruck: Walter Biering $\mathrm{G}$ mbH , G rafischer B etrieb, $M$ ünchen 